\title{
Teaching the Linked Use of Various Softwares for the Simulation of Magnetic Devices
}

\author{
Rosa Ana Salas, Jorge Pleite \\ Departamento de Tecnología Electrónica, Escuela Politécnica Superior, Universidad Carlos III de Madrid \\ Avda. de la Universidad, 30, 28911, Leganés (Madrid), Spain \\ rsalas@ing.uc3m.es
}

\begin{abstract}
The modeling of ferrite inductors is a difficult task due to the nonlinearity of the magnetic fields and the great variety of shapes, sizes of the core and number of turns in the winding. This paper presents a procedure that integrates various standard softwares, modeling and simulation techniques and experimental measurements in order to simulate a soft ferrite inductor for use in a circuit simulator. This procedure is applied to the modeling and simulation of ferrite inductors widely used in the field of power electronics. It can be applied to undergraduate and postgraduate students and help them to understand the behavior of the circuits and the nonlinear physical phenomena involved in power electronics.
\end{abstract}

Index Terms - Core Saturation, Power Losses, Magnetic Devices, Nonlinear Inductors, Ferrite Cores, 2D Finite Element Analysis.

\section{I . Introduction}

Inductors consist of a ferrite core, winding and sometimes a coil former and are available in a great variety of shapes, sizes and number of turns in the winding ${ }^{1-4}$. As an example, Figures 1(a) and 2(a) show two real magnetic components with different geometries, i.e. E and RM type, respectively. The core material shows a nonlinear behavior including saturation, hysteresis and power losses and the magnetic fields are nonlinear, i.e. the spatial distribution of the magnetic fields in the core is inhomogeneous. This inhomogeneity can be seen in Fig. 3 where we show the distribution of the values of the $\mathbf{B}$-field module on the surface of a saturated RM core, displaying a higher concentration on the central column. Therefore, the modeling of these ferrite inductors is a complicated $\operatorname{task}^{5-19}$ as it involves solving nonlinear differential equations which cannot be solved by analytical equations. In order to model them it is necessary to measure the magnetic properties ${ }^{7,8,9,12}$ and use design, simulation and Finite Element Analysis programs ${ }^{14,15,17,18}$.

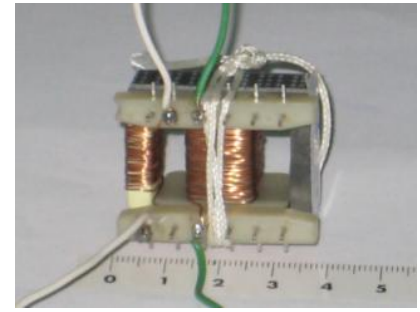

(a)

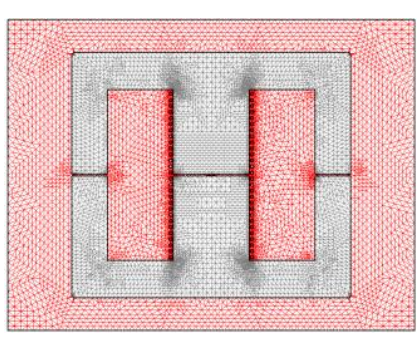

(b)
Fig.1 (a) Real magnetic component with an E core and two windings of 28 turns.(b) Triangular mesh generated by the $2 \mathrm{D}$ simulations.

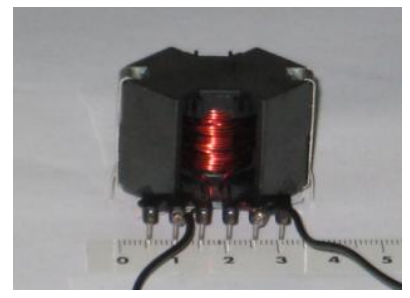

(a)

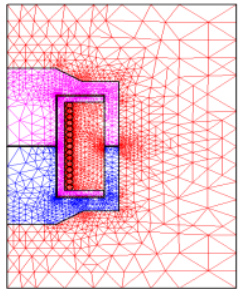

(b)
Fig.2 (a) Real magnetic component with an RM core and two windings of 15 turns. (b) Triangular mesh generated by the $2 \mathrm{D}$ simulations.
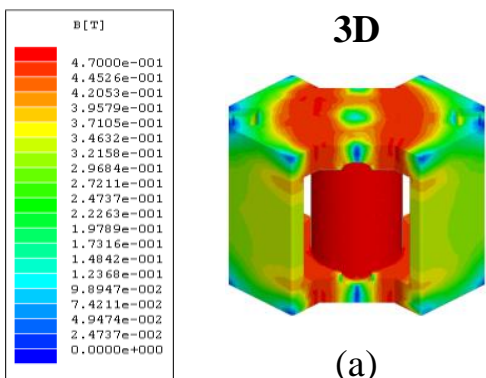

(a)
2D

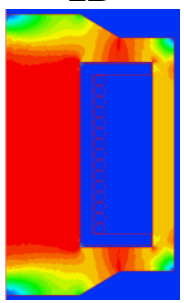

(b)
Fig.3. Values of the $\mathbf{B}$-field module in $2 \mathrm{D}$ and $3 \mathrm{D}$ on the surface of the RM8/I core seen in Fig. 2(a).

In this paper we present a procedure that combines modeling, simulation and experimental measurements on real inductors.

This procedure uses different programming and modeling techniques coupled together: a Computer Aided Design program (AutoCAD), a Finite Element Analysis program (Maxwell), two scientific calculus programs for the numerical solving of derivatives and integrals (Origin and Matlab), a numerical simulation program (Simulink) combined with Matlab and finally an electronic circuit simulation program (PSIM). We demonstrate how in the development of the modeling procedure of ferrites it is necessary to resort to the linked use of different modeling and simulation techniques (finite elements, mathematical equations, circuit simulation, etc...). This procedure is applicable to undergraduate and postgraduate students and helps us to show the students how different softwares can operate together to solve the task of designing and modeling a ferrite inductor. As the procedure is long and complex we have divided it in levels with growing complexity that can be applied to students at different educational stages. 
The paper is organized as follows: In Section II we give a step by step sequence of the procedure. In Section III we show the division of the procedure in levels with growing complexity showing the necessary supplies and softwares. Finally, in Section IV some conclusions are offered.

\section{II . Step by step procedure}

The objective is to obtain the voltage, current and power waveforms of an inductor with a ferrite core corresponding to its serial electric circuit as can be seen in Fig. 4.

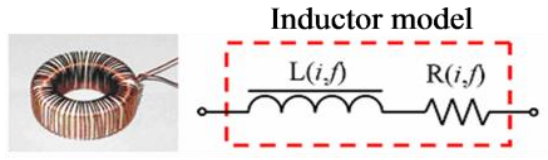

Fig.4 Equivalent Electric Circuit of an inductor with a ferrite core.

It consists of two nonlinear parameters: the inductance $L$ as a function of the excitation current $I$ ( $L-I$ curve) and the resistance $R$ as a function of the rms current and frequency $f$ ( $R-I_{r m s}$ curve). The procedure consists of two different parts: magnetic modeling (computation of the $L-I$ and $R-I_{r m s}$ curves) and electrical modeling through a circuit simulator. The calculation process diagram is shown in Fig. 5.

The process starts with the construction of the inductor that consists of a ferrite core and a coil former on which a copper wire is wound and the measuring of the magnetic parameters under direct current that characterize the ferrite: permeability $\mu$ as a function of the magnetic field $H(\mu-H$ curve) and the magnetic field $B$ as a function of $H$ ( $B-H$ curve). Next, either the 2D (the equivalent section of the ferrite plus its winding and coil former) or the $3 \mathrm{D}$ domain is designed using the program AutoCAD. In Figs. 1(b) and 2(b) we show the $2 \mathrm{D}$ equivalent domains of the real inductors designed in AutoCAD. This design and the magnetic properties are introduced into the Finite Element Analysis program Maxwell. After that, the boundary conditions and excitation current levels are assigned. In order to generate the mesh both in 2D and 3D we chose to carry out an adaptative refinement of the mesh consisting of making a finer mesh at the spatial points where the previously established error level is exceeded (corners, regions with irregular borders, etc..). In each iteration the program computes the magnetic fields, makes an error estimate and refines the mesh. This adaptative meshing reduces the computing time and the convergence and tolerance. This algorithm is implemented into the Maxwell program. In the adaptative procedure the parameters corresponding to the stopping criteria and the percent refinement per pass are introduced. The first ones specify the maximum number of passes and the maximum percent error, also called error tolerance. The percent refinement per pass specifies what percentage of finite elements (triangles in 2D or tetrahedra in 3D) should be refined during each iteration in the initial mesh. In Figs. 1(b) and 2(b) we show the mesh generated in the 2D simulations. As the simulation program's outputs, for each excitation current value we obtain the spatial distribution values of the magnetic fields $\mathbf{B}$ and $\mathbf{H}$ (B-I and $\mathbf{H}$-I Tables) and the $R-I_{r m s}-f$ curve for all working regions of the ferrite from the linear to saturation regions. Using the program Origin and through integration of the $\mathbf{B}$ field, we obtain the $\Phi$ $I$ curve and from this we derive the $L-I$ curve by differentiating $\Phi$ with respect to $I$.

$$
\Phi=\iint_{S} \mathbf{B d} S
$$

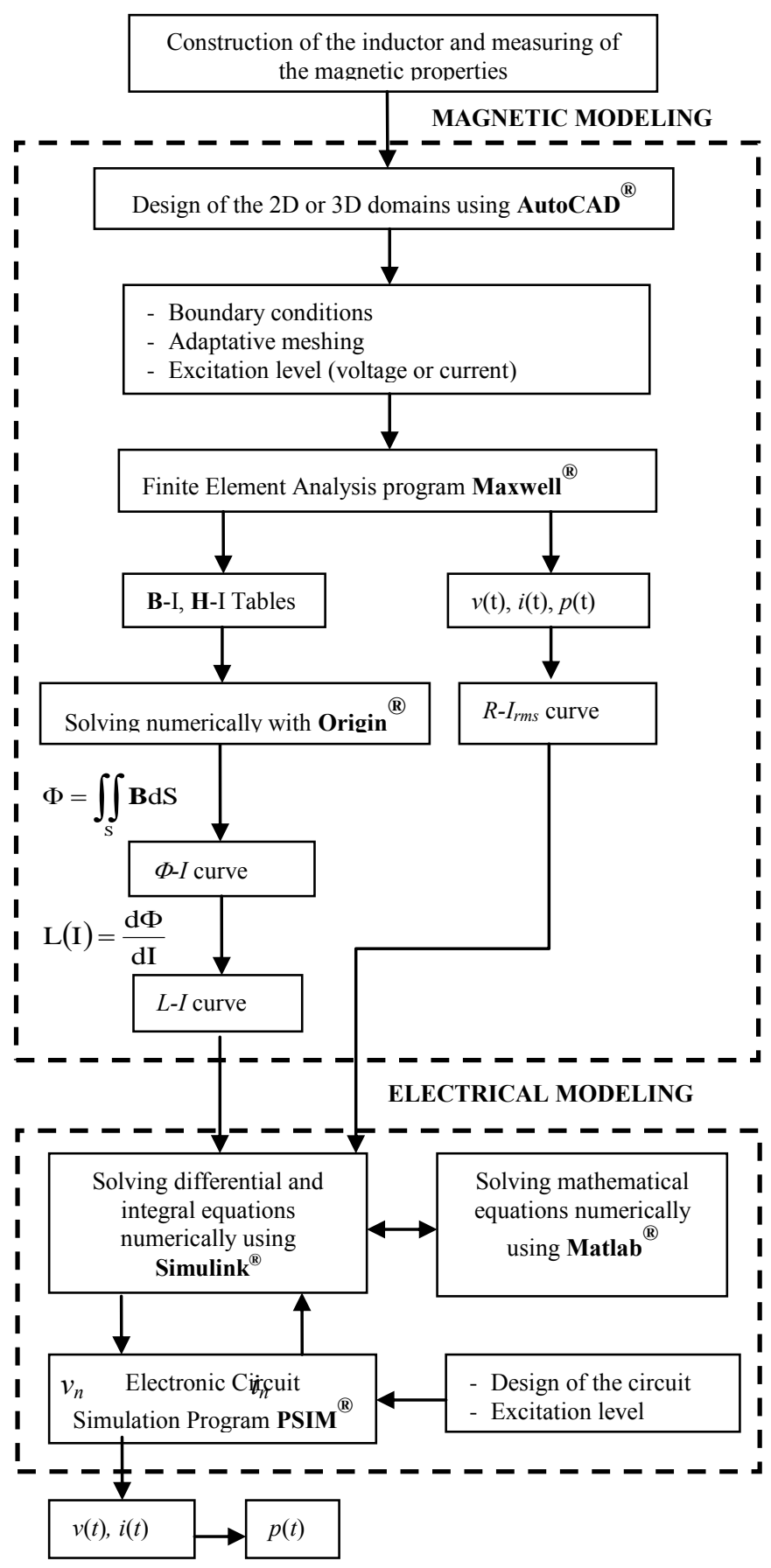

Fig.5 Calculation process diagram. 
The process starts with the construction of the inductor that consists of a ferrite core and a coil former on which a copper wire is wound and the measuring of the magnetic parameters under direct current that characterize the ferrite: permeability $\mu$ as a function of the magnetic field $H(\mu-H$ curve) and the magnetic field $B$ as a function of $H$ ( $B-H$ curve). Next, either the 2D (the equivalent section of the ferrite plus its winding and coil former) or the $3 \mathrm{D}$ domain is designed using the program AutoCAD. In Figs. 1(b) and 2(b) we show the $2 \mathrm{D}$ equivalent domains of the real inductors designed in AutoCAD. This design and the magnetic properties are introduced into the Finite Element Analysis program Maxwell. After that, the boundary conditions and excitation current levels are assigned. In order to generate the mesh both in 2D and 3D we chose to carry out an adaptative refinement of the mesh consisting of making a finer mesh at the spatial points where the previously established error level is exceeded (corners, regions with irregular borders, etc..). In each iteration the program computes the magnetic fields, makes an error estimate and refines the mesh. This adaptative meshing reduces the computing time and the convergence and tolerance. This algorithm is implemented into the Maxwell program. In the adaptative procedure the parameters corresponding to the stopping criteria and the percent refinement per pass are introduced. The first ones specify the maximum number of passes and the maximum percent error, also called error tolerance. The percent refinement per pass specifies what percentage of finite elements (triangles in 2D or tetrahedra in 3D) should be refined during each iteration in the initial mesh. In Figs. 1(b) and 2(b) we show the mesh generated in the 2D simulations. As the simulation program's outputs, for each excitation current value we obtain the spatial distribution values of the magnetic fields $\mathbf{B}$ and $\mathbf{H}$ (B-I and $\mathbf{H}$-I Tables) and the $R-I_{r m s}-f$ curve for all working regions of the ferrite from the linear to saturation regions. Using the program Origin and through integration of the $\mathbf{B}$ field, we obtain the $\Phi$ $I$ curve and from this we derive the $L-I$ curve by differentiating $\Phi$ with respect to $I$.

$$
\Phi=\iint_{S} \mathbf{B d S}
$$

In Figure 6 we show the values of the $\mathbf{B}$-field module in 2D and 3D on the surface of the core computed by the Finite Element Analysis program Maxwell. As can be seen the software has a user friendly and interactive visual interface which makes it interesting for students to use.

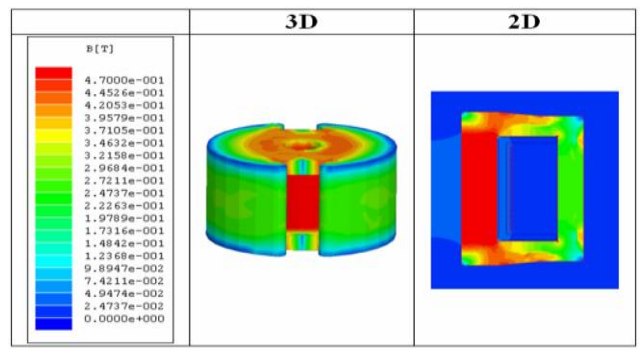

Fig.6 Values of the $\mathbf{B}$-field module in 2D and 3D on the surface of the core.
In Fig. 7 we show an example of an $L-I$ curve and in Fig. 8 an example of an $R-I_{r m s}-f$ curve.

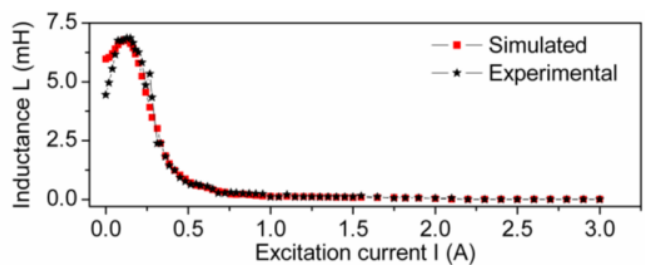

Fig.7 Experimental (stars) and simulated by Finite Element Analysis (squares) $L-I$ curves.

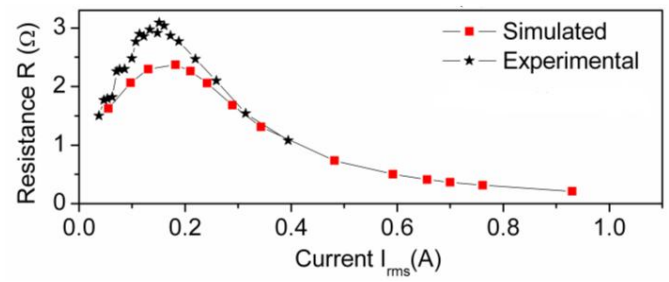

Fig.8 Experimental (stars) and simulated by Finite Element Analysis (squares) $R-I_{r m s}$ curves.

Now we can start the electrical modeling with the joint use of three programs. The $L-I$ and $R-I_{r m s}$ curves are introduced into the Simulink program. With the help of the Matlab program the equation 2 that represents the voltage of the inductor is solved numerically. We draw the electrical circuit to be simulated in the circuit simulator PSIM and assign the voltage and/or current excitation level. At each instant in time the Simulink program sends the excitation current value $i$ that flows through the inductor to PSIM and PSIM sends the voltage $v$ across the inductor to Simulink.

$$
v(t)=L(i, f) \frac{d i}{d t}+i R(i, f)
$$

In Fig. 9 we show an example of an electrical circuit including the nonlinear inductor model excited by a sinusoidal voltage.

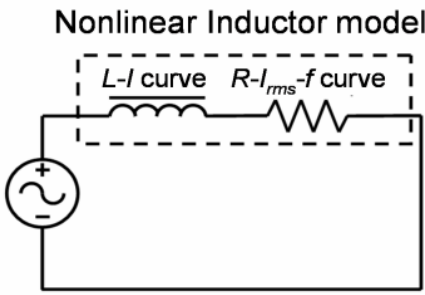

Fig.9 An example of electrical circuit to be drawn in the PSIM program

Finally, we obtain as outputs of the PSIM program the voltage and current waveforms $(v(\mathrm{t}), i(\mathrm{t}))$ and from these data we derive the power waveform $p(\mathrm{t})$ using Origin. In Fig.10 we show an example of these waveforms. The results of Figs. (a), (b) and (c) correspond to the linear region and (d), (e) and (f) to the saturation region. 

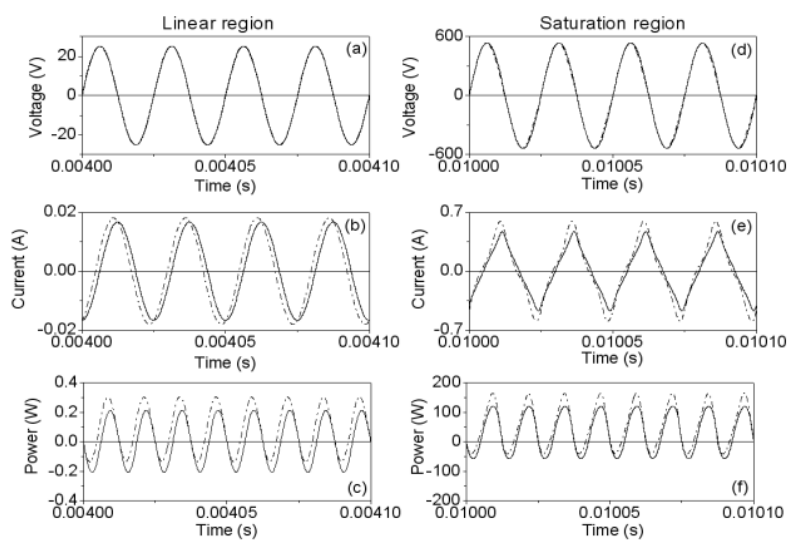

Fig.10 Example of experimental (dotted line) and simulated (solid line) voltage, current and power waveforms for the linear and saturation regions.

\section{Use of the procedure in teaching}

The content of the procedure would be useful for any subject dealing with power electronics that are tought at Universities. As the full procedure is very laborious, we have opted to divide it into four levels with growing complexity.

A. First level

In this level students carry out experimental measurements and analyze the magnetic properties that characterize the ferrites studying their behavior from the linear to the saturation region of the material. This way, already known basic concepts of magnetism are reviewed and used in a practical context. This level consists of four activities: design and construction of the inductors and transformers, preliminary experimental measurements at low current intensity, DC current experiments and AC current experiments. These activities would be useful for undergraduate students and could be suggested as optional additional work for the subject. The optimal organization would be individual or pairwork.

1) First activity of the first level: In this activity the inductors are built by winding copper wire and the necessary number of turns and size of the wire are calculated. The optimal number of turns of the windings is estimated using as parameters the specifications of the measuring equipment, the necessary $B$ and $H$ values to saturate the magnetic core and, in the case of $\mathrm{AC}$ current, the working frequency to be studied. In the case of an inductor excited by a sinusoidal voltage the students would use the following equations in order to derive $N$, being $f$ the frequency, $A_{e}$ the effective cross-sectional area of the core, and $\ell_{e}$ the effective magnetic path length.

$$
\begin{gathered}
V_{p}=2 \pi f N A_{e} B_{p} \\
I_{p}=\frac{H_{p} \ell_{e}}{N} .
\end{gathered}
$$

As the variety of available ferrites is very big, it is possible to study many variables such as different materials, geometries and sizes, diameters of the wire and number of turns. As far as the geometries go, we can study toroidal inductors (no gap) and inductors made with two identical ferrite half-cores (types EE, RM and POT) where the thickness of the air-gap can be varied depending on the applications. Different materials and geometries can be proposed in order to compare the results.

Necessary supplies: enameled copper wire, ferrite cores, and in some cases a coil former.

2) Second activity of the first level: In this activity experimental measurements with an impedance/gain-phase analyzer are made as a function of frequency at low amplitude and linear region to calculate the resonant frequency and the inductance and resistance. Through this experiment the students get to know some characteristics of the inductors as well as the correct range of the working frequencies.

Necessary supplies: previously built inductors, impedance/gain-phase analyzer and a personal computer with the program Labview.

This activity has the advantage of working with a low intensity and therefore not being dangerous for the student.

3) Third activity of the first level: In this activity, experimental measurements are taken under DC current. The objectives of these measurements are to obtain the magnetic properties of the ferrite core ( $B$ - $H$ curve) using a toroidal core and obtain also to obtain the $L-I$ curve of the inductors. In order to obtain the $B$ - $H$ curve, a two-winding toroidal transformer with the same material as the studied ferrite is built and the $\Phi$-I curve is measured for current values from 0 to core saturation. We have chosen a toroidal geometry as it eliminates the effect of the air-gap and the magnetic field lines tend to be circular, thus reducing the edge effect. The $B$ and $H$ values were computed using Matlab as:

$$
\begin{aligned}
& B \approx \frac{1}{N_{s} A_{e}} \int v d t=\frac{\Phi}{N_{s} A_{e}} \\
& H \approx \frac{N_{p} I}{\ell_{e}}
\end{aligned}
$$

where $N_{p}$ and $N_{s}$ are the number of turns of the primary and secondary winding respectively, $A_{e}$ is the effective area of the ferrite core and $\ell_{e}$ is the effective length of the ferrite.

In order to obtain the $L-I$ curve of the inductors, a twowinding transformer with the same core as the studied one is built and the $\Phi-I$ curve is measured for current values from 0 up to core saturation. From this, the $L-I$ curve is derived as:

$$
L(I)=\frac{d \Phi}{d I} .
$$

At this point the students can study the influence of the number of turns and the effect of the air-gap on the $\Phi-I$ and $L-I$ curves by building different transformers and comparing the measurements.

Necessary supplies: ferrite transformers with two windings, enameled copper wire, DC current source, digital 
multimeter, electronic fluxmeter and AC current source.

4) Fourth activity of the first level: In this activity we propose experiments under $\mathrm{AC}$ current by applying an excitation voltage to the ferrite inductors. Experimental measurements were taken with variable voltage at a fixed frequency obtaining a set of voltage and current waveforms. From these results students can compute the $B-H$ hysteresis cycles of the material using the equations (8) and (9). From a saturated hysteresis cycle students can obtain magnetic parameters such as remanence, saturation induction, and coercitive field. Also core losses can be computed calculating the area of the set of cycles. This procedure can be repeated at different frequencies.

$$
\begin{aligned}
& B(t)=\frac{1}{N A_{e}} \int v(t) d t . \\
& H(t) \approx \frac{N i(t)}{\ell_{e}} .
\end{aligned}
$$

This activity might have some risk depending on the voltage and frequency of the experiment.

This activity has been tried at the Carlos III University with undergraduate students with good results. As an example, we show in Figure 11 a set of experimental hysteresis loops of the Ferroxcube $3 \mathrm{~F} 3$ material measured at $20 \mathrm{kHz}$ by the students.

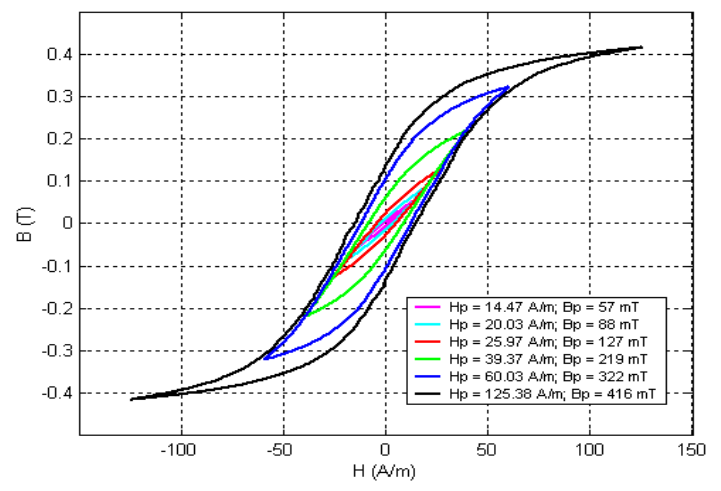

Fig.11 Experimental hysteresis loops of a Ferroxcube 3F3 material measured at $20 \mathrm{kHz}$ for different maximum values of the $\mathrm{H}$ and $\mathrm{B}$ magnetic fields ( $\mathrm{Hp}$, Bp).

\section{B. Second level}

This level focuses on the analysis and simulation of the inductors with ferrite cores using Finite Element Analysis. Through the use of the Maxwell software students can visualize for each excitation current value the flux lines, $\mathbf{B}$ and $\mathbf{H}$ field distributions as shown in Figure 6 and compute from these data the magnetic flux from the linear to the saturation region. This software has a visual interface and provides a physical and extremely useful perspective which helps understand concepts that are difficult for the students. The instructors can propose different geometries and compare 2D to $3 \mathrm{D}$ results.
The procedure without losses (the inductor model consists of an inductance) can be implemented in the PSIM software which in turn works with Matlab and Simulink.

Necessary supplies: Personal computer with the following softwares: AutoCAD to design the inductors, Maxwell software to model, and the PSIM and MatlabSimulink softwares to simulate.

\section{Third level}

This level can be applied to postgraduate students, for example as a final project. Here, the complete procedure is applied to different geommetries and sizes allowing the students to study the influence of the gap-thickness on the magnetic properties. The core losses are included in the procedure (the inductor model consists of inductance and resistance) and the results are validated with experimental measurements for the case of a sinusoidal waveform.

Necessary supplies: Personal computer with the following softwares: AutoCAD to design the inductors, Maxwell software to model, and the PSIM and MatlabSimulink softwares to simulate. Magnetic components and power source to supply the sinusoidal waveform and an oscilloscope to measure the waveforms.

\section{Fourth Level}

This level can be applied to postgraduate students that show interest in research in power electronics. They can carry out all the steps of the procedure (magnetic and electrical modeling) and can validate it for the case of a typical square waveform as is widely used in power electronics. They can verify the complete model of the inductor consisting of the $L-I$ and $R-I_{r m s}$ curves, considering power losses in the core. To validate the waveforms students build a real power converter, for example a DC-DC power converter as shown in Figure 12.

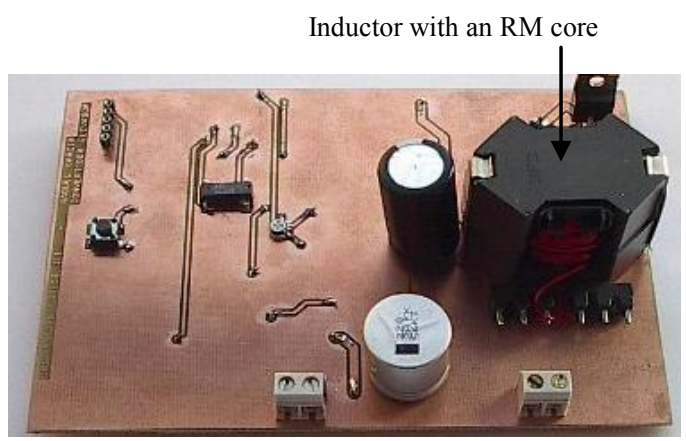

Fig.12 Real DC-DC power converter.

The inductor included in the power converter should be a ferrite core of the same type as the one used in the magnetic modeling, for example an RM ferrite core.

Necessary supplies: Personal computer with the following softwares: AutoCAD to design the inductors, Maxwell software to model, Origin for the numerical calculations and the PSIM and Matlab-Simulink softwares to simulate. Magnetic components and other components to build 
the power converter and an oscilloscope to measure the waveforms.

\section{Conclusions}

Power electronics is a difficult subject for students as it is an interdisciplinary subject by nature, which requires knowledge of digital and analogue electronics as well as of simulation. Apart from this the materials used in power electronics include inductors or transformers with soft ferrite cores that show a nonlinear behavior and involve various variables such as number of turns, geometry and size. Thus the modeling of the ferrite inductors is not an easy task. It is necessary to resort to the measuring of magnetic properties, modeling and simulating techniques and Finite Element programs.

In this paper we have proposed a procedure in which different softwares are used together and which is suited to the modeling the inductors with ferrite cores for use in circuit simulators. This procedure uses different programming and modeling techniques coupled together: a Computer Aided Design program (AutoCAD), a Finite Element Analysis program (Maxwell), two scientific calculus programs for the numerical solving of derivatives and integrals (Origin and Matlab), a numerical simulation program (Simulink) combined with Matlab and finally an electronic circuit simulation program (PSIM). We have divided the paper in two parts. In the first part we have shown our procedure and some illustrative examples. In the second part we have focused on its application to students of different educational stages (undergraduate and postgraduate) and have commented on the required materials. The use of the procedure could motivate the students and help to understand the behavior of the circuits and nonlinear physical phenomena involved in power electronics.

\section{References}

[1] E. C. Snelling. Soft Ferrites, Properties and Applications, Butterworths, London U.K. (1988).

[2] G. E. Fish, Proc. IEEE, 78, 1990.

[3] Ferroxcube, Data Handbook Soft Ferrites and Accessories, 2008, http://ferroxcube.com.

[4] A. Goldman. Magnetic Components for Power Electronics, Springer (2002).

[5] D. C. Jiles and D. L. Atherton, J. Magn. Magn. Mater. 61 (1986).

[6] D. C. Jiles and D. L. Atherton, J. Magn. Magn. Mater. 92 (1990).

[7] V. J. Thottuvelil, T. G. Wilson, H. A. Owen, Jr. IEEE Trans. Magn. 5, 1 (1990).

[8] D. C. Jiles, J. B. Thoelke and M. K. Devine, IEEE Trans. Magn. 28, 1 (1992).

[9] L. Zegadi, J. J. Rousseau, B. Allard, P. Tenant, and D. Renault, IEEE Trans. Magn. 36, 4 (2000).

[10] P. Nakmahachalasint, K. D. T. Ngo, and L. Vu-Quoc, IEEE Trans. Power Electron. 17, 4 (2002).

[11] M. Sippola and R. E. Sepponen, IEEE Trans. Power Electron. 17, 5, (2002).

[12] P. R. Wilson, J. N. Ross, and A. D. Brown, IEEE Trans. Power Electron. 17, 1 (2002).

[13] P. R. Wilson, J. N. Ross, and A. D. Brown, IEEE Trans. Magn. 40, 3 (2004).

[14] D. Lin, P. Zhou, W. N. Fu, Z. Badics, and Z. J. Cendes, IEEE Trans. Magn. 4, 2 (2004)

[15] Y. Zhai and L. Vu-Quoc, IEEE Trans. Magn. 41, 7 (2005).

[16] W. A. Roshen, IEEE Trans. Power Electron. 22, 1 (2007).

[17] R. A. Salas, J. Pleite, E. Olías, and A. Barrado, IEEE Trans. Magn. 44, 7 (2008).

[18] R. A. Salas and J. Pleite, IEEE Trans. Magn. 47, 10 (2011).

[19] D. Zhang and J. E. Fletcher, IEEE Trans. Instrum. Meas. (2012). 